\title{
Quantum non-locality co-exists with locality
}

\author{
Armen E. Allahverdyan and Arshag Danageozian \\ Yerevan Physics Institute, 2 Alikhanian Brothers street, Yerevan 0036, Armenia (a)
}

PACS 03.65.-w-Quantum mechanics

PACS 03.67.-a - Quantum information

\begin{abstract}
Quantum non-locality is normally defined via violations of Bell's inequalities that exclude certain classical hidden variable theories from explaining quantum correlations. Another definition of non-locality refers to the wave-function collapse thereby one can prepare a quantum state from arbitrary far away. In both cases one can debate on whether non-locality is a real physical phenomenon, e.g. one can employ formulations of quantum mechanics that does not use collapse, or one can simply refrain from explaining quantum correlations via classical hidden variables. Here we point out that there is a non-local effect within quantum mechanics, i.e. without involving hidden variables or collapse. This effect is seen via imprecise (i.e. interval-valued) joint probability of two observables, which replaces the ill-defined notion of the precise joint probability for non-commuting observables. It is consistent with all requirements for the joint probability, e.g. those for commuting observales. The non-locality amounts to a fact that (in a two-particle system) the joint imprecise probability of non-commuting two-particle observables (i.e. tensor product of single-particle observables) does not factorize into single-particle contributions, even for uncorrelated states of the two-particle system. The factorization is recovered for a less precise (i.e. the one involving a wider interval) joint probability. This approach to non-locality reconciles it with locality, since the latter emerges as a less precise description.
\end{abstract}

Physics holds signal locality thereby the state of an isolated system (particle) cannot be changed by manip' ulating other particles that interacted with it in the past. Attempts to make this notion stronger created a bunch ' of quantum concepts known as non-locality [1]. Two main aspects of non-locality were identified: (i) applica' tion of the projection postulate to an entangled subsystem that changes (steers) the state of another subsystem arbitrary far away [1]. (ii) Bell's inequalities, their derivation and interpretation [1. Despite of a huge effort devoted to the issue of non-locality, there is no general agreement on whether it really exists [5].

Indeed, (i) does depend on the specific interpretation adopted for quantum mechanics, e.g. it is present in the Copenhagen interpretation, but absent in the many-world interpretation [7],8 and in the consistent histories interpretation [9. Note that the issue of collapse (i.e. what happens to the quantum system after measurement) is not a part of the quantum probability given by Born's rule. The latter can and does apply for describing experiments without worrying about what happens after measurement. This is one reason why the attention in studying non-

(a) To be published in EPL (Europhysics Letters) (2018). locality shifted to Bell's inequalities that do not assume the collapse. Instead they focus on explaining quantum correlations via hidden variables, i.e. they assume structures that are additional with respect to quantum probability. Bell's inequalities can be derived assuming that the hidden variables - in addition to signal locality - hold the outcome independence feature (frequently also called local causality) 2/4. Alternatively, Bell's inequality can be derived without hidden variables, but assuming that frequencies obtained via different non-commuting measurements can be embedded into a single Kolmogorovian space [10. Another version of this condition is that several (more than two) non-commuting quantities do have a joint probability [4, 5]. The joint-probability assumptions can be imposed also on a single quantum particle, where no space-separated sub-systems exist. This leads to single-particle Bell inequalities [11,12. Then, experimental validation of the Bell inequalities show that those additional concepts do not apply to quantum mechanics. Is this the only meaning of non-locality that certain interpretations have a cost to pay, or that specific classic concepts do not apply?

We aim to demonstrate non-locality within quantum 
mechanics without referring to hidden variables, imposed Kolmogorovian probability space or projection postulate (hence it does not depend on which interpretation one prescribes to). The core of this demonstration is that while two non-commuting projectors do not have a joint precise (i.e. usual) probability within quantum mechanics, they do have an imprecise (i.e. interval-valued) joint probability 13, 14. Then non-locality is seen in the fact that the imprecise joint probability operator calculated for tensor products of single-particle (local) observables does not reduce to a tensor product of single-particle operators. Hence the imprecise joint probability (calculated via the ordinary Born's rule) does not reduce to a product of single-particle contributions, even if the state of twoparticle system is not correlated. Our results achieve on the level of imprecise joint statistics what the previous results could not do on the level of precise joint probability for non-commuting observables, since the latter does not exist. This approach reconciles non-locality with locality, because the local (tensor-product based) imprecise joint probability appears to predict a wider interval: it is not wrong (as compared to the non-local one), it is just less precise. We continue by recalling pertinent features of projectors (see 24] for an accessible review), and then the notion of quantum imprecise probability.

Projectors are self-adjoint operators $P$ with $P^{2}=P$. Any projector $P$ in a Hilbert space $\mathcal{H}$ bijectively relates to the sub-space $\mathcal{S}_{P}$ of $\mathcal{H}$ [25]:

$$
\mathcal{S}_{P}=\{|\psi\rangle \in \mathcal{H} ; P|\psi\rangle=|\psi\rangle\} .
$$

Eigenvalues of $P$ are 0 and/or 1 , and it is a quantum analogue of the characteristic function for a classical set 25]. Hence projectors define quantum probability: with a density matrix $\rho$, the probability of finding the eigenvalue 1 of $P$ is given by Born's formula $\operatorname{tr}(\rho P)$.

The simplest projectors are 0 and $I$. For two projectors $P$ and $P^{\prime}$ we define

$$
P \geq P^{\prime} \text { means }\left\langle\psi\left|P-P^{\prime}\right| \psi\right\rangle \geq 0 \text { for any }|\psi\rangle .
$$

Now apply (2) with $|\psi\rangle=\left|\psi_{0}\right\rangle$, where $P\left|\psi_{0}\right\rangle=0$, and then with $|\psi\rangle=\left|\psi_{1}\right\rangle$, where $P^{\prime}\left|\psi_{1}\right\rangle=\left|\psi_{1}\right\rangle$. Hence the eigenvalues of $P$ and $P^{\prime}$ relate to each other leading to

$$
P P^{\prime}=P^{\prime} P=P^{\prime} \text { if } P \geq P^{\prime} .
$$

Projectors (generally non-commuting) support logical operations 25]: negation $P^{\perp}=I-P$, conjunction $P \wedge Q$ $\left(\mathcal{S}_{P \wedge Q}=\mathcal{S}_{Q} \cap \mathcal{S}_{P}\right.$ contains only those vectors that belong both to $\mathcal{S}_{P}$ and $\mathcal{S}_{Q}$ ), and disjunction $P \vee Q$, where $\mathcal{S}_{P \vee Q}$ contains all linear combinations of vectors from $\mathcal{S}_{P}$ and from $\mathcal{S}_{Q}$. There are alternative representations [25]:

$$
\begin{gathered}
P \wedge Q=\max _{R}\left[R \mid R^{2}=R, R \leq P, R \leq Q\right], \\
P \vee Q=\min _{R}\left[R \mid R^{2}=R, R \geq P, R \geq Q\right],
\end{gathered}
$$

where the maximization and minimization go over projectors $R$ [25. Indeed, if $R \leq P$, and $R \leq Q$ in (44), then due to (113), $\mathcal{S}_{R}$ is a subspace of both $\mathcal{S}_{P}$ and $\mathcal{S}_{Q}$. The maximal such subspace is $\mathcal{S}_{P \wedge Q}$. Likewise, if $R \geq P$, and $R \geq Q$, then $\mathcal{S}_{R}$ has to contain both $\mathcal{S}_{P}$ and $\mathcal{S}_{Q}$. The smallest such subspace is $\mathcal{S}_{P \vee Q}$, because joining $\mathcal{S}_{P}$ and $\mathcal{S}_{Q}$ as sets does not result to a linear space.

Now $P \vee Q=0$ only if $P=Q=0$, but $P \wedge Q$ can be zero also for non-zero $P$ and $Q$; e.g. in $\operatorname{dim} \mathcal{H}=2$, we have either $P=Q$ or $P \wedge Q=0$ (and then $P \vee Q=I$ ).

The above three operations are related with each other and with a limiting process [25]:

$$
\begin{array}{r}
P \vee Q=\left(P^{\perp} \wedge Q^{\perp}\right)^{\perp}, \\
P \wedge Q=\lim _{n \rightarrow \infty}(P Q)^{n} .
\end{array}
$$

For $[P, Q] \equiv P Q-Q P=0$ we have from (3 5) the ordinary features of classical characteristic functions

$$
P \wedge Q=P Q, \quad P \vee Q=P+Q-P Q .
$$

Imprecise classical probability generalizes the usual (precise) probabilities [15, 16]: the measure of uncertainty for an event $E$ is an interval $[p(E), \bar{p}(E)]$, where $0 \leq p(E) \leq \bar{p}(E)$ are called lower and upper probabilities, respectively. Now $p(E)($ resp. $1-\bar{p}(E))$ is a measure of a sure evidence in favor (resp. against) of $E$. The event $E$ is surely more probable than $E^{\prime}$, if $p(E) \geq \bar{p}\left(E^{\prime}\right)$. The usual probability is recovered for $p(E)=\bar{p}(E)$. Two different pairs $[p(E), \bar{p}(E)]$ and $\left[\underline{p}^{\prime}(E), \bar{p}^{\prime}(E)\right]$ can hold simultaneously (i.e they are consistent) if

$$
\underline{p}^{\prime}(E) \leq \underline{p}(E) \text { and } \bar{p}^{\prime}(E) \geq \bar{p}(E) .
$$

Every probability is consistent with $\underline{p}^{\prime}(E)=0, \bar{p}^{\prime}(E)=1$. This non-informative situation is not described by the usual theory 15 that inadequately offers for it the homogeneous probability $[15$. Now $[p(E), \bar{p}(E)]$ does not imply that there is an explicit (but possibly unknown) precise probability for $E$ located in between $p(E)$ and $\bar{p}(E)$ [26].

There are various imprecise classical probability models [16 20]. They do have numerous applications e.g. in decision making and artificial intelligence [16. Some of them were applied phenomenologically for describing aspects of the Bell's inequality 21 23. The quantum imprecise probability is to be sought independently, along the physical arguments. Below we recall how it is determined.

Imprecise quatum probability. For two noncommuting projectors $P$ and $Q$ one looks for upper $\bar{\omega}(P, Q)$ and lower $\underline{\omega}(P, Q)$ non-negative probability operators. For a state with density matrix $\rho$, the respective upper and lower probabilities are given by Born's rule:

$$
\bar{p}(P, Q)=\operatorname{tr}(\rho \bar{\omega}(P, Q)), \quad \underline{p}(P, Q)=\operatorname{tr}(\rho \underline{\omega}(P, Q)) .
$$

The linearity of (10) over $\rho$ means that within the standard measurement theory $\bar{p}(P, Q)(p(P, Q))$ can be determined via measuring Hermitean operator $\bar{\omega}(P, Q)(\underline{\omega}(P, Q))$ in a state with an unknown $\rho$ [1]. The following requirements 
determine $\bar{\omega}(P, Q)$ and $\underline{\omega}(P, Q)[13]$

$$
\begin{aligned}
& 0 \leq \underline{\omega}(P, Q)=\underline{\omega}(Q, P) \leq \bar{\omega}(P, Q)=\bar{\omega}(Q, P) \leq I . \\
& {[\omega(P, Q), Q]=[\omega(P, Q), P]=0 \text { for } \omega=\underline{\omega}, \bar{\omega} .} \\
& \underline{\omega}(P, Q)=\bar{\omega}(P, Q)=P Q \quad \text { if } \quad[P, Q]=0 . \\
& \operatorname{tr}(\rho \underline{\omega}(P, Q)) \leq \operatorname{tr}(\rho P Q) \leq \operatorname{tr}(\rho \bar{\omega}(P, Q)) \\
& \text { if }\left[P_{a}, \rho\right]=0 \text { or }[Q b, \rho]=0 .
\end{aligned}
$$

Eqs. (11] 10) forces $0 \leq p(P, Q) \leq \bar{p}(P, Q) \leq 1$ for any $\rho$. Eq. (11) also demands symmetry with respect to $P$ and $Q$ that is necessary for the joint probability.

Let us recall the non-contextuality feature of quantum probability: the ordinary Born's probability $\operatorname{tr}(\rho P)$ is noncontextual, because it does not depend on an observable (Hermitean operator) $A$ whose eigen-projector is $P$. The choice of $A$ is not unique in Hilbert spaces with dimension larger than 2 (different observables having the same eigenprojector need not commute). Now $\underline{\omega}(P, Q)$ and $\bar{\omega}(P, Q)$ are non-contextual in the sense that they depend only on $P$ and $Q$; see also (10). A stronger feature holds: (12) shows that both $\underline{\omega}(P, Q)$ and $\bar{\omega}(P, Q)$ can be measured together with either $P$ or $Q$; cf. (17).

For $[P, Q]=0$ we revert to the usual joint probability; see (13). For $Q=I$ we get from (13, 10) the marginal and precise probability of $P$. Eqs. (14) 15) also refer to the consistency [in the sense of (9)] with the precise probability, because the latter is well-defined not only for $[P, Q]=0$, but also under conditions (15), where it amounts to $\operatorname{tr}(\rho P Q)$. The latter can be calculated as an average $\operatorname{tr}\left(\rho \frac{P Q+Q P}{2}\right)$ of the Hermitean operator $\frac{P Q+Q P}{2}$.

Eqs. (11] 14) suffice for deducing [13]:

$\underline{\omega}(P, Q)=P \wedge Q, \quad \bar{\omega}(P, Q)=P \vee Q-(P-Q)^{2}$,

where (16) are (resp.) the largest and the smallest positive operators holding (11) 14). Now $\underline{\omega}(P, Q)$ is a projector, while $\bar{\omega}(P, Q)$ is generally just a non-negative operator. For $[P, Q]=0$ we deduce (13) from (8) and (16).

Eqs. (16) imply (12), because - as follows from (4. 5) and checked directly- $P \wedge Q, P \vee Q$ and $(P-Q)^{2}$ commute with each other and with $P$ and $Q$. Hence

$$
[\bar{\omega}(P, Q), \underline{\omega}(P, Q)]=0 .
$$

The origin of (16) is understood from (11, 12) and (4) 5), i.e. $P \wedge Q$ and $P \vee Q$ qualify as certain (resp.) lower and upper probability operators, while the factor $(P-Q)^{2}$ in (16) is needed to ensure (13).

An important geometric feature of (16) is that both $P Q P$ (i.e. the restriction of $Q$ into $\mathcal{S}_{P}$ ) and $Q P Q$ hold:

$$
\underline{\omega}(P, Q) \leq P Q P, Q P Q \leq \bar{\omega}(P, Q) .
$$

Now $\underline{\omega}(P, Q) \leq P Q P$ is shown from $P \wedge Q \leq Q$ [see (44)] that implies $P \wedge Q=P(P \wedge Q) P \leq P Q P$.
And $P Q P \leq \bar{\omega}(P, Q)$ follows from: $\bar{\omega}(P, Q)-P Q P=$ $\bar{\omega}(P, Q)-P \bar{\omega}(P, Q) P=\bar{\omega}(P, Q)(I-P) \geq 0$ recalling that $[\bar{\omega}(P, Q), P]=0$.

Eq. (11) can be deduced from (16, 18). The latter also implies a version of sub- and super-additivity for $\bar{\omega}(P, Q)$ and $\underline{\omega}(P, Q)$, respectively:

$$
\sum_{a} \bar{\omega}\left(P_{a}, Q\right) \geq Q, \quad \sum_{a} \underline{\omega}\left(P_{a}, Q\right) \leq Q
$$

where $\sum_{a} P_{a}=I$. Hence $\underline{\omega}(P, Q)$ and $\bar{\omega}(P, Q)$ do not lead to additive probabilities: the additive marginalization can still be applied, but it leads to an upper bound $\sum_{a} \bar{\omega}\left(P_{a}, Q\right)$ (and lower bound $\sum_{a} \underline{\omega}\left(P_{a}, Q\right)$ ) for the correct marginal probability $\bar{\omega}(I, Q)=\underline{\omega}(I, Q)$; see (19). The correct marginals are calculated from (13) by taking $P=I$ or $Q=I$. Note that monotonicity does not hold, i.e. generally $\bar{\omega}(P, Q) \not \leq \bar{\omega}(I, Q)=Q$, though $P \leq I$.

Eq. (18) directly leads to (14, 15). The following feature is seen from (16)

$$
U \omega(P, Q) U^{\dagger}=\omega\left(U P U^{\dagger}, U Q U^{\dagger}\right), \quad \omega=\underline{\omega}, \bar{\omega},
$$

where $U$ is a unitary operator: $U U^{\dagger}=I$. For further features of the imprecise probability see [13,14. Ref. 14] shows that it is also consistent with the quantum conditional (two-time) probability. We emphasize that (16) are respectively the minimal and maximal operators holding (10 15). If some of those conditions are omitted, the imprecise probabilities can only become more precise.

Imprecise probability for combined systems. We now turn to applying the above formalism for two particles denoted by indices 1 and 2, living resp. in Hilbert spaces $\mathcal{H}_{1}$ and $\mathcal{H}_{2}$. Let $P_{k}$ and $Q_{k}$ be two generally non-commuting $\left(\left[P_{k}, Q_{k}\right] \neq 0\right)$ projectors living in $\mathcal{H}_{k}$, $k=1,2$. This is the standard set-up for designing and studying Bell's inequalities [4. For two (non-identical) particle these projectors read

$$
P_{1} \otimes I, \quad Q_{1} \otimes I, \quad I \otimes P_{2}, \quad I \otimes Q_{2} .
$$

Projectors refering to different particles naturally commute, e.g. $\left[P_{1} \otimes I, I \otimes P_{2}\right]=0$. Hence the joint feature is described by the projector $\left(P_{1} \otimes I\right)\left(I \otimes P_{2}\right)=P_{1} \otimes P_{2}$, which - being a tensor product of single-particle operators - can be measured separately in sub-systems (and then bringing the measurement data together). For factorized states, $\rho_{1} \otimes \rho_{2}$, these measurements lead to uncorrelated probabilities.

There are two ways (resp. (22) and (23)) for looking at the joint statistics of two local operators:

$$
\begin{array}{ll}
\underline{\omega}\left(P_{1} \otimes P_{2}, Q_{1} \otimes Q_{2}\right), & \bar{\omega}\left(P_{1} \otimes P_{2}, Q_{1} \otimes Q_{2}\right) ; \\
\underline{\omega}\left(P_{1} \otimes Q_{2}, Q_{1} \otimes P_{2}\right), & \bar{\omega}\left(P_{1} \otimes Q_{2}, Q_{1} \otimes P_{2}\right) .
\end{array}
$$

For the lower probability operators in (22, 23), (7) leads to an intuitively expected outcome:

$$
\begin{aligned}
\underline{\omega}\left(P_{1} \otimes P_{2}, Q_{1} \otimes Q_{2}\right) & =\underline{\omega}\left(P_{1}, Q_{1}\right) \otimes \underline{\omega}\left(P_{2}, Q_{2}\right) \\
& =\underline{\omega}\left(P_{1} \otimes Q_{2}, Q_{1} \otimes P_{2}\right) .
\end{aligned}
$$


Eq. (24) shows that the lower probability operator is tensor product of two local projectors. Eq. (25) confirms that two different ways (22) and (23) of combining commuting projectors leads to the same outcome. Hence the lower probability is local.

The situation with the upper probability is different. Our main result (deduced in Appendix) is formulated simpler after joint block-diagonalization of $P_{k}$ and $Q_{k}$ (for each $k=1,2)$ via a suitable unitary $U_{k}$ [cf. (20)]. This is known as the CS-representation [27 29]:

$$
\begin{aligned}
& P_{k}=\operatorname{dg}\left[\hat{P}_{k}^{\left[2 m_{k}\right]}, I^{\left[m_{k}(1)\right]}, 0^{\left[m_{k}(2)\right]}, I^{\left[m_{k}(3)\right]}, 0^{\left[m_{k}(4)\right]}\right], \\
& Q_{k}=\operatorname{dg}\left[\hat{Q}_{k}^{\left[2 m_{k}\right]}, I^{\left[m_{k}(1)\right]}, I^{\left[m_{k}(2)\right]}, 0^{\left[m_{k}(3)\right]}, 0^{\left[m_{k}(4)\right]}\right],
\end{aligned}
$$

where $\operatorname{dg}[A, B, \ldots]$ means block-diagonal matrix with block $A, B, \ldots$ Upper indices are integers that indicate the dimension of each square block matrix, e.g. $I^{\left[m_{k}(2)\right]}$ means $m_{k}(2) \times m_{k}(2)$ unity matrix. We omit these indices, whenever their implications are clear from the context. According to (26. 27), the original Hilbert space $\mathcal{H}$ can be represented as a direct sum of subspaces with dimensions $2 m_{k}, m_{k}(1), m_{k}(2), m_{k}(3)$ and $m_{k}(4)$. Some of them can be zero, i.e. the subspaces will be absent from (26, 27).

We recall a possible explicit representation for the noncommuting parts $\hat{P}_{k}$ and $\hat{Q}_{k}$ of (resp.) $P_{k}$ and $Q_{k}$ as $2 m_{k} \times 2 m_{k}$ matrices 2729 :

$$
\hat{P}_{k}=\left(\begin{array}{cc}
C_{k}^{2} & C_{k} S_{k} \\
C_{k} S_{k} & S_{k}^{2}
\end{array}\right), \hat{Q}_{k}=\left(\begin{array}{cc}
I & 0 \\
0 & 0
\end{array}\right),
$$

where $C_{k}$ and $S_{k}$ are invertible, self-adjoint $m_{k} \times m_{k}$ matrices holding

$$
C_{k}^{2}+S_{k}^{2}=I, \quad\left[C_{k}, S_{k}\right]=0 .
$$

The simplest example of (26 29) are the projectors for $x$ and $z$-components of two spin- $\frac{1}{2}$ particles. Here $\operatorname{dim} \mathcal{H}_{k}=$ 2 , only the subspace with $2 m_{k}=2$ is present, and $\hat{P}_{k}=$ $\frac{1+\sigma_{x}}{2}, \hat{Q}_{k}=\frac{1+\sigma_{z}}{2}$, where $\sigma_{x}$ and $\sigma_{z}$ are the Pauli matrices. Hence $C_{k}=S_{k}=\frac{1}{\sqrt{2}}$ reduce to numbers.

The following relations are deduced from (28, 29)

$$
\hat{P}_{k} \vee \hat{Q}_{k}=I, \quad \hat{P}_{k} \wedge \hat{Q}_{k}=0, \quad \operatorname{tr} \hat{P}_{k}=\operatorname{tr} \hat{Q}_{k}=m_{k} .
$$

Hence in all other subspaces besides $\hat{P}_{k} \vee \hat{Q}_{k}=I$ [cf. (26)] ], the projectors $P_{k}$ and $Q_{k}$ commute.

In the representation (26, 27), the upper and lower probability operators (16) read via (30)

$$
\begin{array}{r}
\bar{\omega}\left(P_{k}, Q_{k}\right)=\operatorname{dg}\left(I-\left(\hat{P}_{k}-\hat{Q}_{k}\right)^{2}, I, 0,0,0\right), \\
\underline{\omega}\left(P_{k}, Q_{k}\right)=\operatorname{dg}(0, I, 0,0,0) .
\end{array}
$$

Here is our main result obtained within the CS representation (26, 27) (see Appendix):

$$
\begin{aligned}
& \bar{\omega}\left(P_{1}, Q_{1}\right) \otimes \bar{\omega}\left(P_{2}, Q_{2}\right)-\bar{\omega}\left(P_{1} \otimes P_{2}, Q_{1} \otimes Q_{2}\right) \\
& =\operatorname{dg}\left(\bar{\omega}\left(\hat{P}_{1}^{\perp} \otimes \hat{Q}_{2}^{\perp}, \hat{Q}_{1}^{\perp} \otimes \hat{P}_{2}^{\perp}\right), 0, \ldots, 0\right) \geq 0,
\end{aligned}
$$

where other 24 blocks nullify, and the inequality follows from the definition (16) of $\bar{\omega}$.

Eq. (34) shows that the upper probability operator $\bar{\omega}\left(P_{1} \otimes P_{2}, Q_{1} \otimes Q_{2}\right)$ does not reduce to the tensor product $\bar{\omega}\left(P_{1}, Q_{1}\right) \otimes \bar{\omega}\left(P_{2}, Q_{2}\right)$, i.e. it does not have the local form. We take this as indications of non-locality. This feature is stronger than the notion of entanglement, since it is formulated without density matrices, i.e. on the level of probability operators. Now $\bar{\omega}\left(P_{1} \otimes P_{2}, Q_{1} \otimes Q_{2}\right)=\bar{\omega}\left(P_{1}, Q_{1}\right) \otimes$ $\bar{\omega}\left(P_{2}, Q_{2}\right)$ is recovered whenever at least one pair commutes, i.e. either $\left[P_{1}, Q_{1}\right]=0$ or $\left[P_{2}, Q_{2}\right]=0$ holds; e.g. $\left[P_{1}, Q_{1}\right]=0$ implies that $\hat{P}_{1}$ and $\hat{Q}_{1}$ are absent from (34). Hence $\bar{\omega}\left(P_{1} \otimes P_{2}, Q_{1} \otimes Q_{2}\right) \neq \bar{\omega}\left(P_{1}, Q_{1}\right) \otimes \bar{\omega}\left(P_{2}, Q_{2}\right)$ is due to non-commutativity at both 1 and 2 . In particular, we get (as we should) the correct marginal upper probability $\bar{\omega}\left(P_{1}, Q_{1}\right) \otimes I$ for the particle 1 whenever $P_{2}=Q_{2}=I$.

The inequality in (34) means that the local form $\bar{\omega}\left(P_{1}, Q_{1}\right) \otimes \bar{\omega}\left(P_{2}, Q_{2}\right)$ of the upper probability is less precise than the non-local expression $\bar{\omega}\left(P_{1} \otimes P_{2}, Q_{1} \otimes Q_{2}\right)$, i.e. upper probabilities calculated via $\operatorname{tr}\left(\rho \bar{\omega}\left(P_{1}, Q_{1}\right) \otimes\right.$ $\left.\bar{\omega}\left(P_{2}, Q_{2}\right)\right)$ will (for an arbitrary density matrix) be larger than those calculated $\operatorname{tr}\left(\rho \bar{\omega}\left(P_{1} \otimes P_{2}, Q_{1} \otimes Q_{2}\right)\right)$. In this sense, the imprecise probability reconciles locality with non-locality.

Eqs. (34) and (22, 23) imply

$$
\bar{\omega}\left(P_{1} \otimes Q_{2}, Q_{1} \otimes P_{2}\right)-\bar{\omega}\left(P_{1} \otimes P_{2}, Q_{1} \otimes Q_{2}\right) \neq 0 .
$$

Hence depending on how we combine commuting projectors - via (22) or (23) - we shall get different upper probability operators. This clearly contrasts with features 24 25) of the lower probability operator. As seen below, (35) is generally non-zero even if we calculate the upper probabilities (10) for independent subsystems, i.e. via tensorproduct states $\rho=\rho_{1} \otimes \rho_{2}$.

Two spin- $1 / 2$ particles. We now illustrate 334 35. via a pair of spin- $1 / 2$ particles, where $m_{k}=1$ in (26, 27), and also $m_{k}(1)=m_{k}(2)=m_{k}(3)=m_{k}(4)=0$ for $k=$ 1,2 . For simplicity we also assume $P_{1}=P_{2}=P$ and $Q_{1}=$ $Q_{2}=Q$ (the index $k$ drops out), and $C_{k}=S_{k}=1 / \sqrt{2}$ in (28, 291), i.e. $P$ and $Q$ amount to $x$ and $z$ components of the spin $\frac{1}{2}$. In (34) we employ $P \vee Q=(P+Q)(P+Q)^{-}$ [30, where $X^{-}$is the pseudo-inverse of matrix $X$ [31. We use definition (41) for the tensor product. The result reads from (33) or from (34):

$$
\begin{aligned}
& \bar{\omega}(P, Q) \otimes \bar{\omega}(P, Q)-\bar{\omega}(P \otimes P, Q \otimes Q) \\
& =\bar{\omega}(P \otimes Q, Q \otimes P)=\frac{1}{12}\left(\begin{array}{cccc}
0 & 0 & 0 & 0 \\
0 & 2 & -1 & -1 \\
0 & -1 & 2 & -1 \\
0 & -1 & -1 & 2
\end{array}\right), \\
& \bar{\omega}(P, Q) \otimes \bar{\omega}(P, Q)-\bar{\omega}(P \otimes Q, Q \otimes P) \\
& =\bar{\omega}(P \otimes P, Q \otimes Q)=\frac{1}{12}\left(\begin{array}{cccc}
1 & -1 & -1 & 0 \\
-1 & 1 & 1 & 0 \\
-1 & 1 & 1 & 0 \\
0 & 0 & 0 & 3
\end{array}\right) .
\end{aligned}
$$


Now both (36) and (37) have the same eigenvalues: 0 and $1 / 4$ (both doubly degenerate). However, they do not commute. Let us define the following separable state:

$$
\rho \otimes \rho, \quad \rho=\left(\begin{array}{cc}
a & b e^{i \phi} \\
b e^{-i \phi} & 1-a
\end{array}\right),
$$

where $\rho$ is a one-particle density matrix with real parameters $a, b$ and $\phi$ that hold $1 \geq a \geq 0$ and $a(1-a) \geq b^{2}$. These conditions ensure $\rho \geq 0$. The difference between (37) and (36) is not zero even for separable states:

$$
\begin{aligned}
& \operatorname{tr}[(\bar{\omega}(P \otimes P, Q \otimes Q)-\bar{\omega}(P \otimes Q, Q \otimes P)) \rho \otimes \rho]= \\
& \frac{1}{12}\left[(1-2 a)^{2}+4 b^{2}+4 b(1-2 a) \cos (\phi)\right] \geq 0 .
\end{aligned}
$$

For separable states (39) has a definite sign. No definite sign is possible for all states, since $\operatorname{tr}[\bar{\omega}(P \otimes P, Q \otimes Q)-\bar{\omega}(P \otimes Q, Q \otimes P)]=0$ due to the fact that $\bar{\omega}(P \otimes P, Q \otimes Q)$ and $\bar{\omega}(P \otimes Q, Q \otimes P)$ have the same eigenvalues.

Summary. We studied the joint statistics of noncommuting observables shared between two particles. This is a standard set-up for defining quantum nonlocality. Upon assuming the existence of joint probabilities for non-commuting observables (or alternatively, specific features of hidden variable theories), it leads to the Bell inequalities for certain (entangled) states 4. However, the precise joint probability for non-commuting observables is denied by quantum mechanics [4,14, restricting the message of Bell inequalities to inapplicability of certain non-quantum concepts to quantum mechanics.

In contrast, we addressed the above set-up from the viewpoint of imprecise joint probability for noncommuting observables. This concept does exist: it is well-defined and holds all requirements asked by quantum mechanics from a joint probability. Our basic message is that for making more precise predictions for the joint probability of two-particle observables requires nonlocality, because the corresponding upper probability is not a tensor product of one-particle factors. In contrast to other forms of quantum non-locality (e.g. the non-locality without entanglement [32]), the uncovered form of nonlocality is formulated on the level of observables, i.e. independently from the notion of quantum states. Hence it survives for separable states, as we saw for the simplest example.

Appendix. For deriving (34), we introduce two different types of tensor products:

$$
A \otimes B \quad \text { and } \quad A \bullet B=\overline{A \otimes B}=B \otimes A,
$$

where $A \otimes B$ is the usual from left-to-right tensor (Kroenecker) product, e.g. when $A$ is a $2 \times 2$ matrix,

$$
\left(\begin{array}{ll}
a_{11} & a_{12} \\
a_{21} & a_{22}
\end{array}\right) \otimes B=\left(\begin{array}{ll}
a_{11} B & a_{12} B \\
a_{21} B & a_{22} B
\end{array}\right),
$$

where $a_{i k} B$ (with $i, k=1,2$ ) means that a number $a_{i k}$ is multiplied over all matrix elements of $B$. We stress that
$A \otimes B$ and $A \bullet B$ in (40) are related by a unitary operator that does not depend on $A$ and $B$. Now we note

$$
\begin{aligned}
& \overline{\operatorname{dg}(A, B) \otimes \operatorname{dg}(C, D)}=\operatorname{dg}(U, V) \times \\
& \operatorname{dg}(A \otimes \operatorname{dg}(C, D), B \otimes \operatorname{dg}(C, D)) \operatorname{dg}\left(U^{\dagger}, V^{\dagger}\right) \\
& =\operatorname{dg}(A \bullet \operatorname{dg}(C, D), B \bullet \operatorname{dg}(C, D)) \\
& =\operatorname{dg}(A \bullet C, A \bullet D, B \bullet C, B \bullet D),
\end{aligned}
$$

where when going from (42) to (43) we used (41), and where (44) is achieved from (43) by means of blockdiagonal unitary matrices with blocks $U$ and $V$. Eq. (45) is a block-diagonal matrix that will be useful below.

For $A$ and $B$ being projectors we get

$$
\begin{aligned}
& \operatorname{dg}(A, B) \wedge \operatorname{dg}(C, D)=\operatorname{dg}(A \wedge C, B \wedge D), \\
& \operatorname{dg}(A, B) \vee \operatorname{dg}(C, D)=\operatorname{dg}(A \vee C, B \vee D),
\end{aligned}
$$

where (46) is derived from (7), while (47) is deduced from (46) via (6). Eqs. (42 47) straightforwardly generalize to an arbitrary number of blocks. Now recall from (20) that

$$
\begin{aligned}
& \left.\overline{\bar{\omega}\left(P_{1} \otimes P_{2}, Q_{1} \otimes Q_{2}\right)}=\bar{\omega} \overline{\left(P_{1} \otimes P_{2}\right.}, \overline{Q_{1} \otimes Q_{2}}\right) \\
& =\overline{P_{1} \otimes P_{2}} \vee \overline{Q_{1} \otimes Q_{2}}-\left(\overline{P_{1} \otimes P_{2}}-\overline{Q_{1} \otimes Q_{2}}\right)^{2} .
\end{aligned}
$$

Using (42 45) and (26 27) we write down

$$
\begin{array}{ll}
\overline{P_{1} \otimes P_{2}}= & \operatorname{dg}\left(\hat{P}_{1} \bullet \hat{P}_{2}, \hat{P}_{1}, 0, \hat{P}_{1}, 0, \ldots\right) \\
\overline{Q_{1} \otimes Q_{2}}= & \operatorname{dg}\left(\hat{Q}_{1} \bullet \hat{Q}_{2}, \hat{Q}_{1}, \hat{Q}_{1}, 0,0, \ldots\right)
\end{array}
$$

where for simplicity we write $\hat{P}_{1} \bullet I$ as $\hat{P}_{1}$ and $I \bullet I$ as $I$, and where other 20 blocks (in each equation) were omitted for simplicity. Now both $\overline{P_{1} \otimes P_{2}} \vee \overline{Q_{1} \otimes Q_{2}}$ and $\left(\overline{P_{1} \otimes P_{2}}-\overline{Q_{1} \otimes Q_{2}}\right)^{2}$ are easy to calculate, since they respect the block-diagonal structure; cf. (47). Here we, in particular, employ $\left(\hat{P}_{1} \bullet I\right) \vee\left(\hat{Q}_{1} \bullet I\right)=I \bullet I=I$; see the first equation in (30). Hence

$$
\begin{aligned}
& \overline{P_{1} \otimes P_{2}} \vee \overline{Q_{1} \otimes Q_{2}}= \\
& \operatorname{dg}\left(\hat{P}_{1} \bullet \hat{P}_{2} \vee \hat{Q}_{1} \bullet \hat{Q}_{2}, I, \hat{Q}_{1}, \hat{P}_{1}, 0, \ldots\right), \\
& \left.\overline{\left(P_{1} \otimes P_{2}\right.}-\overline{Q_{1} \otimes Q_{2}}\right)^{2}= \\
& \operatorname{dg}\left(\left(\hat{P}_{1} \bullet \hat{P}_{2}-\hat{Q}_{1} \bullet \hat{Q}_{2}\right)^{2},\left(\hat{P}_{1}-\hat{Q}_{1}\right)^{2}, \hat{Q}_{1}, \hat{P}_{1}, 0, \ldots\right)
\end{aligned}
$$

Employing (31) we work out in the same way

$$
\begin{aligned}
& \overline{\bar{\omega}\left(P_{1}, Q_{1}\right) \otimes \bar{\omega}\left(P_{2}, Q_{2}\right)}= \\
& \operatorname{dg}\left(\left(I-\left(\hat{P}_{1}-\hat{Q}_{1}\right)^{2}\right) \bullet\left(I-\left(\hat{P}_{2}-\hat{Q}_{2}\right)^{2}\right),\right. \\
& \left.I-\left(\hat{P}_{1}-\hat{Q}_{1}\right)^{2}, 0,0,0, \ldots\right) .
\end{aligned}
$$

These intermediate formulas lead finally to

$$
\begin{aligned}
& \overline{\bar{\omega}\left(P_{1} \otimes P_{2}, Q_{1} \otimes Q_{2}\right)}-\overline{\bar{\omega}\left(P_{1}, Q_{1}\right) \otimes \bar{\omega}\left(P_{2}, Q_{2}\right)} \\
& =\operatorname{dg}\left(\left(\hat{P}_{1} \bullet \hat{P}_{2}\right) \vee\left(\hat{Q}_{1} \bullet \hat{Q}_{2}\right)-\left(\hat{P}_{1} \bullet \hat{P}_{2}-\hat{Q}_{1} \bullet \hat{Q}_{2}\right)^{2}\right. \\
& \left.-\left(I-\left(\hat{P}_{1}-\hat{Q}_{1}\right)^{2}\right) \bullet\left(I-\left(\hat{P}_{2}-\hat{Q}_{2}\right)^{2}\right), 0, \ldots, 0\right),
\end{aligned}
$$


where the last 24 blocks are zero. Once only one block is non-zero, we obtain

$$
\begin{aligned}
& \bar{\omega}\left(P_{1} \otimes P_{2}, Q_{1} \otimes Q_{2}\right)-\bar{\omega}\left(P_{1}, Q_{1}\right) \otimes \bar{\omega}\left(P_{2}, Q_{2}\right) \\
& =\operatorname{dg}\left(\left(\hat{P}_{1} \otimes \hat{P}_{2}\right) \vee\left(\hat{Q}_{1} \otimes \hat{Q}_{2}\right)-\left(\hat{P}_{1} \otimes \hat{P}_{2}-\hat{Q}_{1} \otimes \hat{Q}_{2}\right)^{2}\right. \\
& \left.-\left(I-\left(\hat{P}_{1}-\hat{Q}_{1}\right)^{2}\right) \otimes\left(I-\left(\hat{P}_{2}-\hat{Q}_{2}\right)^{2}\right), 0, \ldots, 0\right) .
\end{aligned}
$$

Now (55) can be simplified by employing

$$
\begin{aligned}
& \left(\hat{P}_{1}^{\perp} \otimes \hat{Q}_{2}^{\perp}-\hat{Q}_{1}^{\perp} \otimes \hat{P}_{2}^{\perp}\right)^{2}=I-\left(\hat{P}_{1} \otimes \hat{P}_{2}-\hat{Q}_{1} \otimes \hat{Q}_{2}\right)^{2} \\
& -\left(I-\left(\hat{P}_{1}-\hat{Q}_{1}\right)^{2}\right) \otimes\left(I-\left(\hat{P}_{2}-\hat{Q}_{2}\right)^{2}\right),
\end{aligned}
$$

whose derivation is algebraically tedious, but straightforward. Below we shall prove that

$$
\left(\hat{P}_{1} \otimes \hat{P}_{2}\right) \vee\left(\hat{Q}_{1} \otimes \hat{Q}_{2}\right)+\left(\hat{P}_{1}^{\perp} \otimes \hat{Q}_{2}^{\perp}\right) \vee\left(\hat{Q}_{1}^{\perp} \otimes \hat{P}_{2}^{\perp}\right)=I .
$$

Using (56, 57) in (55) finishes the proof of (34).

The proof of (57) is consists of three steps. First, we note that the two projectors in left-hand-side of (57) are orthogonal to each other thanks to

$$
\begin{gathered}
\left(\hat{P}_{1} \otimes \hat{P}_{2}\right)\left(\hat{P}_{1}^{\perp} \otimes \hat{Q}_{2}^{\perp}\right)=\left(\hat{Q}_{1} \otimes \hat{Q}_{2}\right)\left(\hat{P}_{1}^{\perp} \otimes \hat{Q}_{2}^{\perp}\right)=0 \\
\left(\hat{P}_{1} \otimes \hat{P}_{2}\right)\left(\hat{Q}_{1}^{\perp} \otimes \hat{P}_{2}^{\perp}\right)=\left(\hat{Q}_{1} \otimes \hat{Q}_{2}\right)\left(\hat{Q}_{1}^{\perp} \otimes \hat{P}_{2}^{\perp}\right)=0 .
\end{gathered}
$$

Hence the left-hand-side of (57) is $\leq I$. Second, we get using (7) 30)

$$
\begin{aligned}
& \left(\hat{P}_{1} \otimes \hat{P}_{2}\right) \wedge\left(\hat{Q}_{1} \otimes \hat{Q}_{2}\right)=\left(\hat{P}_{1} \wedge \hat{Q}_{1}\right) \otimes\left(\hat{P}_{2} \wedge \hat{Q}_{2}\right) \\
& =\left(\hat{P}_{1}^{\perp} \otimes \hat{Q}_{2}^{\perp}\right) \wedge\left(\hat{Q}_{1}^{\perp} \otimes \hat{P}_{2}^{\perp}\right)=0 .
\end{aligned}
$$

Third, recall that if $\mathcal{S}_{P}$ is the Hilbert space generated by a projector $P$, then $\operatorname{dim}\left[\mathcal{S}_{P}\right]=\operatorname{tr} P$. Now aply to both sides of (57) the known formula 29]

$$
\operatorname{tr}(P \vee Q)+\operatorname{tr}(P \wedge Q)=\operatorname{tr}(P)+\operatorname{tr}(Q),
$$

that holds for any projectors $[P, Q] \neq 0$, and use $(58600)$ :

$$
\begin{aligned}
& \operatorname{tr}\left(\hat{P}_{1} \otimes \hat{P}_{2}\right)=\operatorname{tr}\left(\hat{Q}_{1} \otimes \hat{Q}_{2}\right)=m_{1} m_{2}, \\
& \operatorname{tr}\left(\left(\hat{P}_{1} \otimes \hat{P}_{2}\right) \vee\left(\hat{Q}_{1} \otimes \hat{Q}_{2}\right)\right) \\
= & \operatorname{tr}\left(\left(\hat{P}_{1}^{\perp} \otimes \hat{Q}_{2}^{\perp}\right) \vee\left(\hat{Q}_{1}^{\perp} \otimes \hat{P}_{2}^{\perp}\right)\right)=2 m_{1} m_{2} .
\end{aligned}
$$

Eqs. 62, 63) show that the traces of both sides (=projectors) of (57) are equal. Hence (57) was proved.

\section{REFERENCES}

[1] W.M. de Muynck, Foundations of quantum mechanics, an empiricist approach (Kluwer Academic Publishers, Dordrecht, 2002).

[2] J. Jarrett, Nous, 18, 569-589 (1984).

[3] J. Pienaar, Found. Phys. 46, 104119 (2016).

[4] A. Fine, Phys. Rev. Lett. 48, 291 (1982). J. Math. Phys. 23, 1306 (1982).
[5] M. Zukowski and C. Brukner, J. Phys. A: Math. Theor. 47, 424009 (2014)

[6] D. Deutsch, Proc. R. Soc. Lond. A 468, 531544 (2011).

[7] F.J. Tipler, PNAS, 111, 11281-11286 (2014).

[8] G. Bacciagaluppi, in T. Placek and J. Butterfield, eds. Non-locality and Modality (Kluwer, Dordrecht, 2002), p. 105.

[9] R.B. Griffiths, Found. Phys. 41, 705 (2011).

[10] P.H. Eberhard, Nuovo Cimento B, 38, 75 (1977).

[11] S. P. Gudder, Found. Phys. 14, 997 (1984).

[12] L. Accardi and A. Fedullo, Lett. Nuovo Cimento 34, 161 (1982).

[13] A.E. Allahverdyan, New J. Phys. 17, 085005 (2015).

[14] A.E. Allahverdyan and A. Danageozian, Phys. Rev. A 97, 030102(R) (2018).

[15] FAQs of Imprecise Probability, available at https://msse.gatech.edu/research/interval/

WhyImpreciseProbability.html

[16] Introduction to Imprecise Probabilities ed. by T. Augustin, F.P.A. Coolen, G. de Cooman and M.C.M. Troffaes (J. Wiley \& Sons, 2014).

[17] P.J. Huber, Robust Statistics (Wiley \& Sons, NY, 1981).

[18] P. Suppes and M. Zanotti, Erkenntnis, 31, 323-345 (1989).

[19] A.P. Dempster, Ann. Math. Stat. 38, 325 (1967).

[20] G. Shafer, The Mathematical Theory of Evidence (Princeton University Press, Princeton 1976).

[21] P. Suppes and M. Zanotti, Found. Phys. 21, 1479 (1991).

[22] A. Vourdas, J. Math. Phys. 55, 082107 (2014).

[23] B.H. Feintzeig and S.C. Fletcher, Found. Phys. 47, 294 (2017)

[24] C. G. Adler and J. F. Wirth, Am. J. Phys. 51, 412 (1983).

[25] J.M. Jauch, Foundations of Quantum Mechanics (Addison-Wesley, Reading, 1968).

[26] A. Papamarcou and T.L. Fine, The Annals of Probability, 14, 710-723 (1986).

[27] J. Dixmier, Rev. Sci. 86, 387 (1948).

[28] P. Halmos, Transactions of the American Mathematical Society, 144, 381 (1969).

[29] G. M. Hardegree, Found. Phys. 7, 495 (1977)

[30] R. Piziak, P. L. Odell and R. Hahn, Computers and Mathematics with Applications 37, 67 (1999).

[31] If $X=U D U^{\dagger}$, where $U$ is unitary and $D$ is diagonal with elemenents $\left(d_{1}, . ., d_{n}, 0, \ldots, 0\right)$, where $\left(d_{1}, . ., d_{n}\right)$ are strictly non-zero, then $X^{-}=U D^{-} U^{\dagger}$, where $D^{-}$is a diagonal matrix with elements $\left(1 / d_{1}, . ., 1 / d_{n}, 0, \ldots, 0\right)$.

[32] C. H. Bennett et al., Phys. Rev. A 59, 1070 (1999). 